\title{
Beta-Ketothiolase Deficiency
}

National Cancer Institute

\section{Source}

National Cancer Institute. Beta-Ketothiolase Deficiency. NCI Thesaurus. Code C98841.

A rare autosomal recessive inherited disorder caused by mutations in the ACAT 1 gene. It is characterized by the reduction or elimination of the enzyme mitochondrial acetoacetylCoA thiolase which is responsible for the metabolism of the amino acid isoleucine and ketone-body metabolism. Signs and symptoms appear early in life and include vomiting, dehydration, breathing difficulties, seizures, lethargy, and coma. 